\section{Placebo-Pflaster lindert chronische Schmerzen}

\author{
Bei Patienten mit chronischen muskuloskelettalen Schmerzen lassen sich mit- \\ hilfe eines Placebopräparates, appliziert als transdermales Pflaster, die \\ Schmerzen lindern. Eine Konditionierung verbessert dagegen das Ergebnis \\ bei akuten Schmerzen nicht.
}

P lacel acebowirkungen sind bisher bei Schmerzpatienten am besten erforscht. Man weiß zwar, dass bei Gesunden positive Erfahrungen den analgetischen Effekt verstärken können. Unklar war jedoch bisher, wie sich bei Schmerzpatienten frühere Erfahrungen auswirken. Deshalb haben Neuropsychologen und Schmerzforscher um Prof. Dr. Herta Flor und Maike Müller vom Zentralinstitut für Seelische Gesundheit in Mannheim untersucht, wie bei Patienten mit chronischen Schmerzen mündliche Informationen, Lernen und frühere Therapieerfahrungen die Stärke der analgetischen Wirkung von Placebo beeinflussen. Sie stellten die Hypothese auf, dass bereits der mündliche Hinweis auf die Anwendung eines potenten Analgetikums eine entsprechende Wirkung des Placebos bei akuten experimentellen und chronischen Schmerzen erzielt.

\section{Patienten hatten chronische muskuloskelettale Schmerzen}

An der Studie nahmen 49 Patienten zwischen 18 und 75 Jahren teil, die seit mindestens sechs Monaten chronische muskuloskelettale Schmerzen hatten. Den Teilnehmern der Therapiegruppe wurde gesagt, dass in der Studie das Schmerzempfinden unter dem Einfluss eines stark wirksamen transdermal per Pflaster verabreichten Analgetikums erforscht werde. Darüber hinaus wurden sie mündlich darauf hingewiesen, dass die Intervention einen stark schmerzlindernden Effekt habe. Sie erhielten ein Pflaster, das auf den Rücken geklebt wurde und mit einem Beutel physiologischer Kochsalzlösung verbunden war. Daran angeschlossen war ein weiterer Beutel mit der Aufschrift „Taroxin-hydromorphone, $1 \mathrm{ml}=10 \mathrm{mg}^{\text {“. Den Studi- }}$ enteilnehmern wurde gesagt, obwohl das Pflaster auf den Rücken geklebt worden sei, wirke das Medikament zentral. Innerhalb von fünf Minuten lindere es sowohl chronische als auch akute
Schmerzen „sehr effektiv“. Die Schmerzstärke mussten die Patienten anhand der visuellen Analogskala bewerten.

Die Hälfte der Patienten in der Behandlungsgruppe erhielt zudem eine klassische Konditionierung: Nach der Applikation des pharmakologischen Placebos wurde die Stärke eines Druckschmerzstimulus nach und nach verringert. In einer Gruppe ohne Behandlung $(\mathrm{n}=10)$ wurde auf die Konditionierung verzichtet. Vor dem Experiment wurden alle Teilnehmer zur bisherigen Erfahrung mit einer Schmerztherapie befragt. Der Erfolg (besser oder schlechter als vorher) sollte mithilfe der Sieben-Punkte-Skala nach Likert beziffert werden.

\section{Konditionierung hatte keinen Einfluss}

Wie die Wissenschaftler berichten, wurde bei Patienten, die auf das pharmakologische Placebo ansprachen, die Stärke des experimentell induzierten akuten Schmerzes um $29 \%$ verringert, und zwar mit einer großen Effektstärke nach Cohen von 1,47. Der Parameter Effektstärke ist eine Alternative zur statistischen Signifikanz und ein Maß für die Größe des Zusammenhangs. Mit dem Ergebnis werden frühere Untersuchungsergebnisse bestätigt. Bei der Beurteilung der chronischen Schmerzen konnte eine Reduktion um $40 \%$ dokumentiert werden bei einer Effektstärke von 1,40. Zum Vergleich: In Studien zum Rückenschmerz wurden in den Placebogruppen Reduktionen zwischen $8 \%$ und $60 \%$ beobachtet. Wurden in der aktuellen Studie alle 49 Patienten in die statistische Auswertung einbezogen, beobachteten die Wissenschaftler eine Schmerzreduktion um immer noch $28 \%$ bei einer Effektstärke von 0,78. Die Konditionierung verstärkte allerdings nicht die durch Erwartung induzierte Wirksamkeit des Placebos, wie es in früheren Untersuchungen bei Gesunden beobachtet worden war.

Schließlich ergab die Befragung der Patienten, dass diejenigen, die früher negative Erfahrungen mit einer Schmerztherapie hatten, stärker auf die Placeboanalgesie ansprachen als Patienten mit positiver Erfahrung in der Anamnese. Die zunächst widersprüchliche Beobachtung erklären sich die Wissenschaftler damit, dass Patienten, die oft schlechte Erfahrungen mit gängiger Schmerztherapie gesammelt hatten, am meisten von einem neuen, potenziell wirksameren Therapieansatz profitieren. Möglicherweise beruht dies auf der Erwartungshaltung der Patienten.

(ple)

Müller M et al. Treatment history and placebo responses to experimental and clinical pain in chronic pain patients. Eur J Pain 2016; online 7. April; doi: 10.1002/ejp.877

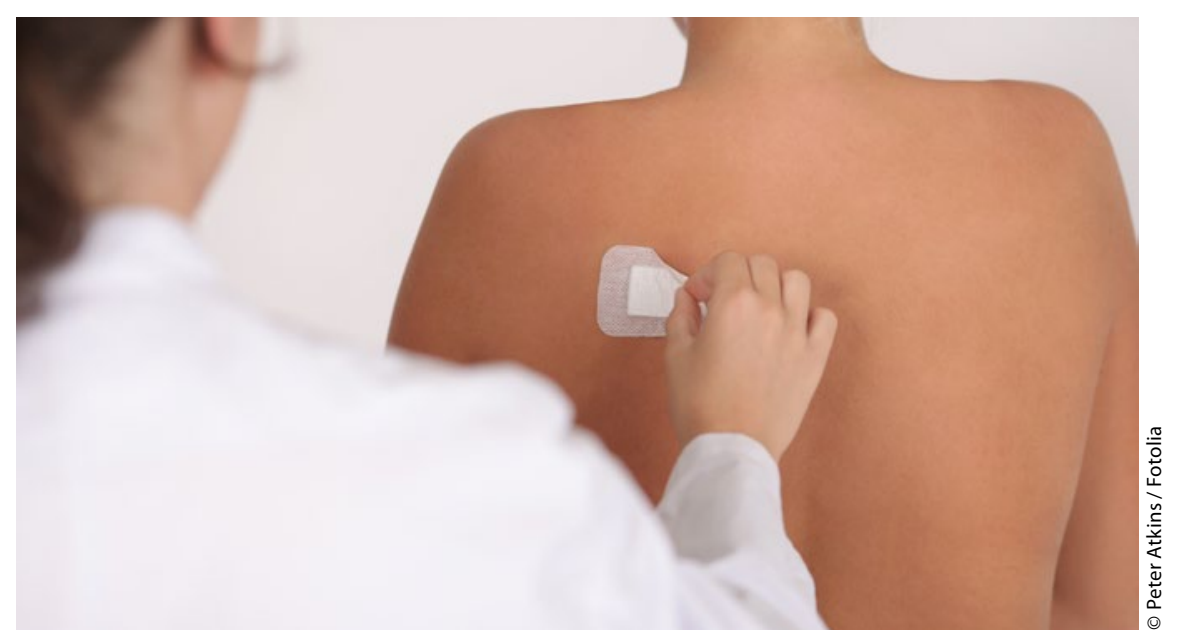

Schon die Ankündigung eines Analgetikum hilft bei der Schmerzlinderung. 